\title{
Competitive displacement of full-length HIV-1 Nef from the Hck SH3 domain by a high-affinity artificial peptide
}

\author{
Thomas Stangler,1,a, Tuyen Tran',2,a,b, Silke \\ Hoffmann ${ }^{1,2}$, Holger Schmidt',2, Esther Jonas ${ }^{1,2}$ \\ and Dieter Willbold ${ }^{1,2, *}$ \\ 1 Institut für Physikalische Biologie and BMFZ, Heinrich- \\ Heine-Universität Düsseldorf, Universitätsstraße 1, \\ D-40225 Düsseldorf, Germany \\ ${ }^{2}$ Institut für Neurowissenschaften und Biophysik INB-2, \\ Forschungszentrum Jülich, D-52425 Jülich, Germany \\ ${ }^{*}$ Corresponding author \\ e-mail: d.willbold@fz-juelich.de
}

\begin{abstract}
We studied the interaction of the artificial 12-aa prolinerich peptide PD1 with the SH3 domain of the hematopoietic cell kinase Hck and the peptide's potency in competitively displacing HIV-1 Nef from the Hck $\mathrm{SH} 3$ domain. PD1 was obtained from a phage display screen and exhibits exceptional affinity for the Hck $\mathrm{SH} 3$ domain $\left(K_{d}=0.23 \mu \mathrm{M}\right)$. Competition experiments using NMR spectroscopy demonstrate that the peptide even displaces Nef from Hck SH3 and allow for estimation of the Nef-Hck SH3 dissociation constant $\left(K_{\mathrm{d}}=0.44 \mu \mathrm{M}\right)$, the strongest $\mathrm{SH} 3$ ligand interaction known so far. Consequences of this study for novel antiviral concepts are discussed.
\end{abstract}

Keywords: competition; Hck; HIV; Nef; NMR; proteinpeptide interaction; $\mathrm{SH}$.

The viral Nef protein plays a pivotal role in human immunodeficiency virus (HIV) pathogenesis. Nef is incorporated into virus particles (Welker et al., 1996) and administers a variety of functions during HIV infection, whereby it interferes with several cellular processes. Well-known effects of Nef are the down-modulation of CD4 and $\mathrm{MHCl}$ (Garcia and Miller, 1991; Aiken et al., 1994; Greenberg et al., 1998; Mangasarian et al., 1999) from the cell surface. In addition, Nef down-modulates CD28 (Bell et al., 2001; Swigut et al., 2001), CXR4 (Hrecka et al., 2005), CD8 $\alpha \beta$ (Stove et al., 2005) and TCRCD3 (Schaefer et al., 2002). Nef has dramatic effects on virus load and pathogenicity in vivo (Kestler et al., 1991; Schindler et al., 2006). Long-term survival of a group of HIV-infected patients could be attributed to a defective nef gene (Deacon et al., 1995; Kirchhoff et al., 1995).

a These authors contributed equally to this work.

b Present address: Institut für Biochemie, Westfälische WilhelmsUniversität Münster, Wilhelm-Klemm-Str. 2, D-48149 Münster, Germany.
Nef interacts with a variety of cellular signal transduction proteins such as various cytoplasmic protein tyrosine kinases (PTKs) (reviewed by Renkema and Saksela, 2000). A typical modular protein unit of PTKs involved in protein-protein interactions is the approximately 60-aa Src homology 3 (SH3) domain. A PXXP proline repeat motif (where $X$ is any amino acid) has been identified as the minimal consensus sequence defining the interaction motif for SH3 domain-interacting proteins and peptides. HIV Nef sequences exhibit an evolutionarily conserved PXXP motif, which was shown to be involved in the interaction with PTKs and is required for higher in vitro replicative potential of $\mathrm{Nef}^{+}$viruses (Saksela et al., 1995; Briggs et al., 1997; Craig et al., 1999). Crystallographic studies of the core domain of Nef in complex with Fyn SH3 domains (Lee et al., 1996; Arold et al., 1997) have shown the importance of the PXXP motif in interaction, and also underlined the contribution of tertiary interactions to binding affinity. The $\mathrm{SH} 3$ domain of hematopoietic-cell kinase Hck exhibits the highest known affinity for Nef (Lee et al., 1995). The Hck SH3 interaction is regarded as the prototype of Nef-SH3 domain interaction (Saksela, 2004). Cell-based assays demonstrate that binding to Nef leads to Hck kinase activation and subsequent deregulation of growth signaling (Briggs et al., 1997; Moarefi et al., 1997). Hck is expressed in macrophages, which are only a subset of infected cells, but macrophages play an important role in HIV infection and in the development of AIDS (Balter, 1996). In a very recent study, Hck could be identified as an in vivo effector of Nef signaling, which makes Hck a potential target for anti-HIV drug discovery (Trible et al., 2006). Here we report on our investigations of the Nef-Hck SH3 interaction and its competition by a short artificial peptide. We used NMR spectroscopy, which is a powerful tool for studying protein-ligand interactions (Lepre et al., 2004; Stangler et al., 2006), in combination with fluorescence spectroscopy for quantitative analysis of the Nef-Hck SH3-PD1 interaction.

We recently identified the artificial peptide PD1 with the amino acid sequence acetyl-HSKYPLPPLPSL-amide as a ligand to the human lymphocyte-specific kinase Lck SH3 domain (Tran et al., 2005). PD1 shows an unusual ligand sequence for $\mathrm{SH} 3$ binding in type I orientation because it lacks the typical basic anchor residue at position $\mathrm{P}_{-3}$. It does, however, have a lysine residue at position $\mathrm{P}_{-4}$. The recently published solution structure of the Hck SH3 complex structure with PD1 indeed suggests that the lysine at position $\mathrm{P}_{-4}$ adopts the function of the anchor residue (Schmidt et al., 2007). Peptide PD1 contains the core consensus LPx(L/A)P motif described for $\mathrm{SH} 3$ class I' ligands. As expected, PD1 binds to HckSH3 
in type I orientation. PD1 residues 5-10 adopt a class I' binding conformation, which is defined by the SH3-II conformation of the W113 side chain in the solution structure of the HckSH3:PD1 complex. Peptide residues Leu6-Pro10 adopt a PPII helix conformation with typical $\phi$ and $\psi$ angles. The four $\mathrm{N}$-terminal PD1 residues attach to the SH3 surface in an 'S'-shaped fashion, generating various interactions of hydrophobic and electrostatic origin. The PD1 Tyr-4 side chain, which corresponds to the typical anchor position $\mathrm{P}_{-3}$, covers a hydrophobic pocket and is involved in extensive van der Waals' interactions with the imidazole ring of $\mathrm{Hck} \mathrm{SH} 3 \mathrm{His}-92$. The side chain of Lys-3, positioned at $\mathrm{P}_{-4}$, points towards the compass pocket of HckSH3.

We also investigated this peptide for binding to the Hck $\mathrm{SH} 3$ domain. PD1 was purchased as a reversed-phase HPLC-purified and mass spectrometry-confirmed product from JPT Peptide Technologies (Berlin, Germany). Hck SH3 domain (HckSH3; aa 60-140) was purified as previously described (Tran et al., 2005). Using SH3domain intrinsic tryptophan fluorescence, we carried out fluorescence titration experiments with PD1 to determine the affinity of the HckSH3-PD1 interaction (Figure 1A). The estimated dissociation constant $K_{\mathrm{d}}$ for HckSH3-PD1 interaction is $0.23 \pm 0.03 \mu \mathrm{M}$. We also investigated the interaction of HckSH3 and PD1 by NMR methods. Figure $2 \mathrm{~A}$ shows an overlay of ${ }^{1} \mathrm{H}-{ }^{15} \mathrm{~N}$ HSQC spectra (Bodenhausen and Ruben, 1980) of $0.26 \mathrm{mM} \mathrm{HckSH} 3$ in the absence and presence of equimolar PD1. During ongoing titration of HckSH3 with PD1, resonances of free HckSH3 exhibited decreasing peak intensities upon addition of
PD1. On the other hand, new resonances appeared upon addition of PD1, which correspond to HckSH3 resonances in the PD1-liganded state. This indicates that dissociation of PD1 from HckSH3 is slow on the NMR time scale, which agrees well with the dissociation constant of $0.23 \mu \mathrm{M}$.

In a similar manner, we investigated the interaction of HckSH3 and Nef by NMR methods. Full-length $\mathrm{Nef}_{2-210}$ (HIV-1 isolate SF2, accession no. P03407), starting with residue Gly-2, was expressed in E. coli BL21 Rosetta (DE3) cells harboring plasmid pUbi-Nef as a poly-histidine tagged (His-tagged) ubiquitin-fused construct in a pTKK19xb/ub vector (Kohno et al., 1998). The fusion protein was purified using a Ni-NTA metal affinity column (Qiagen, Hilden, Germany). The His-tagged ubiquitin part was cleaved off by His-tagged ubiquitin hydrolase, yielding pure $\mathrm{Nef}_{2-210}$ without any modification after another Ni-NTA metal affinity run. The identity of the $\mathrm{Nef}_{2-210}$ protein was confirmed by mass spectrometric peptide mapping. Degassed buffers containing a total $\mathrm{NaCl}$ concentration of $300 \mathrm{~mm}$ and $14 \mathrm{~mm}$ 2-mercaptoethanol were used. Figure 2B shows the ${ }^{1} \mathrm{H}-{ }^{15} \mathrm{~N}$ HSQC NMR spectra of $0.18 \mathrm{~mm}$ HckSH3 in the presence of $0.42 \mathrm{~mm}$ Nef. Most resonances of free HckSH3 (compare Figure 2A) disappeared upon Nef addition, which is a clear indication of Nef-HckSH3 interaction. This behavior is most likely explained by dynamic processes (self-interaction or conformational exchange in the HckSH3-Nef complex) for these resonances on time scales very unfavorable for NMR spectroscopy. This behavior of Nef-HckSH3 is not surprising, since full-length Nef exhibits unfavorable
A

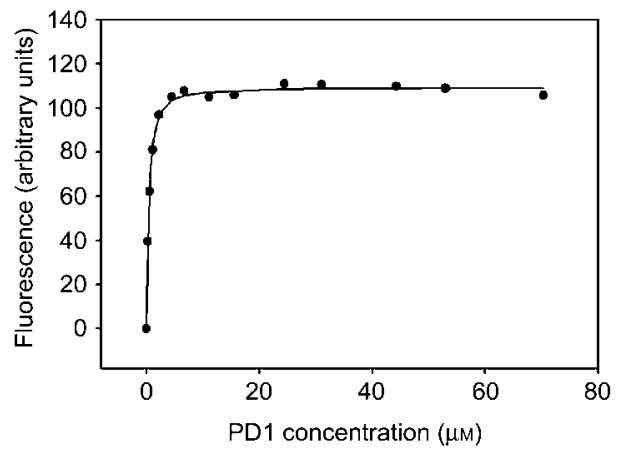

B

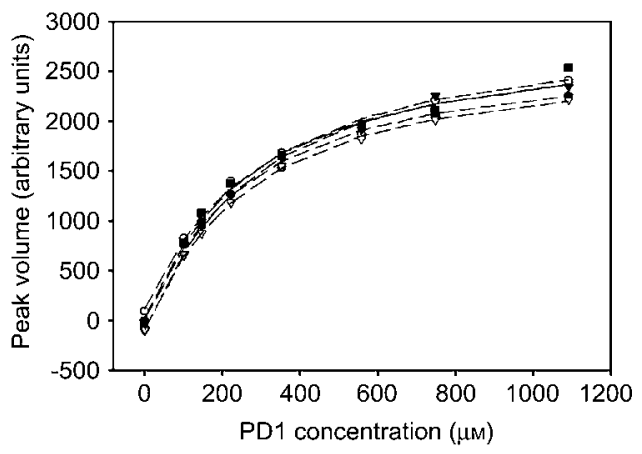

Figure 1 PD1 binding to HckSH3 demonstrated by fluorescence and NMR spectroscopy.

(A) PD1 peptide interacts with HckSH3. Fluorescence titration of $0.5 \mu \mathrm{M}$ HckSH3 with PD1 peptide. The fluorescence signal (@) is shown as a function of PD1 peptide concentration. Fluorescence measurements were carried out at room temperature on a PerkinElmer LS55 fluorescence spectrometer using excitation and emission wavelengths of 290 and $345 \mathrm{~nm}$, respectively. PD1 peptide in PBS was added in small increments to $0.5 \mu \mathrm{M} \mathrm{HckSH3}$ in the same buffer. Values result from the fluorescence of HckSH3 in the presence of the indicated concentration of PD1 peptide in comparison to a buffer control containing the corresponding concentration of tyrosinamide. Assuming a simple bimolecular interaction between HckSH3 and PD1 peptide, the data were described by a model solely based on the law of mass action, which accounts for ligand depletion (Tran et al., 2005). Non-linear curve fitting was carried out to fit the model to the experimental data and to obtain the dissociation constant $K_{\mathrm{d}}=0.23 \pm 0.03 \mu \mathrm{M}$ (solid line).

(B) Quantitative evaluation of competitive NMR titration of $0.18 \mathrm{~mm} \mathrm{HckSH3}$ in the presence of $0.42 \mathrm{~mm}$ Nef with PD1 peptide. Five resonance signals marked in Figure 2B were used for quantitative evaluation. For these signals, the volume of two-dimensional NMR

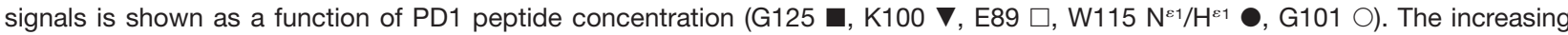
volumes indicate Nef displacement and are a measure of the HckSH3-PD1 complex population. For a quantitative description of the NMR competition data, we used a model describing the competition of ligand (Nef) and inhibitor (PD1) for the same binding site on the target protein $(\mathrm{HckSH} 3)$, solely based on the law of mass action and accounting for ligand or inhibitor depletion [Wang, 1995; Eqs. (8)-(11) in Lepre et al., 2004]. Non-linear curve fitting (dashed lines) allows for estimation of the Nef-HckSH3 dissociation constant if the PD1-HckSH3 dissociation constant is known $\left(K_{d}=0.23 \mu \mathrm{M}\right)$. The Nef-HckSH3 dissociation constant was estimated for the five aforementioned independent resonance signals, resulting in an average value of $0.44 \pm 0.03 \mu \mathrm{M}$. The model allows for reliable determination of $\mathrm{IC}_{50}$, which was determined to be $0.25 \pm 0.02 \mathrm{mM}$ PD1 in the presence of $0.42 \mathrm{~mm}$ Nef. 

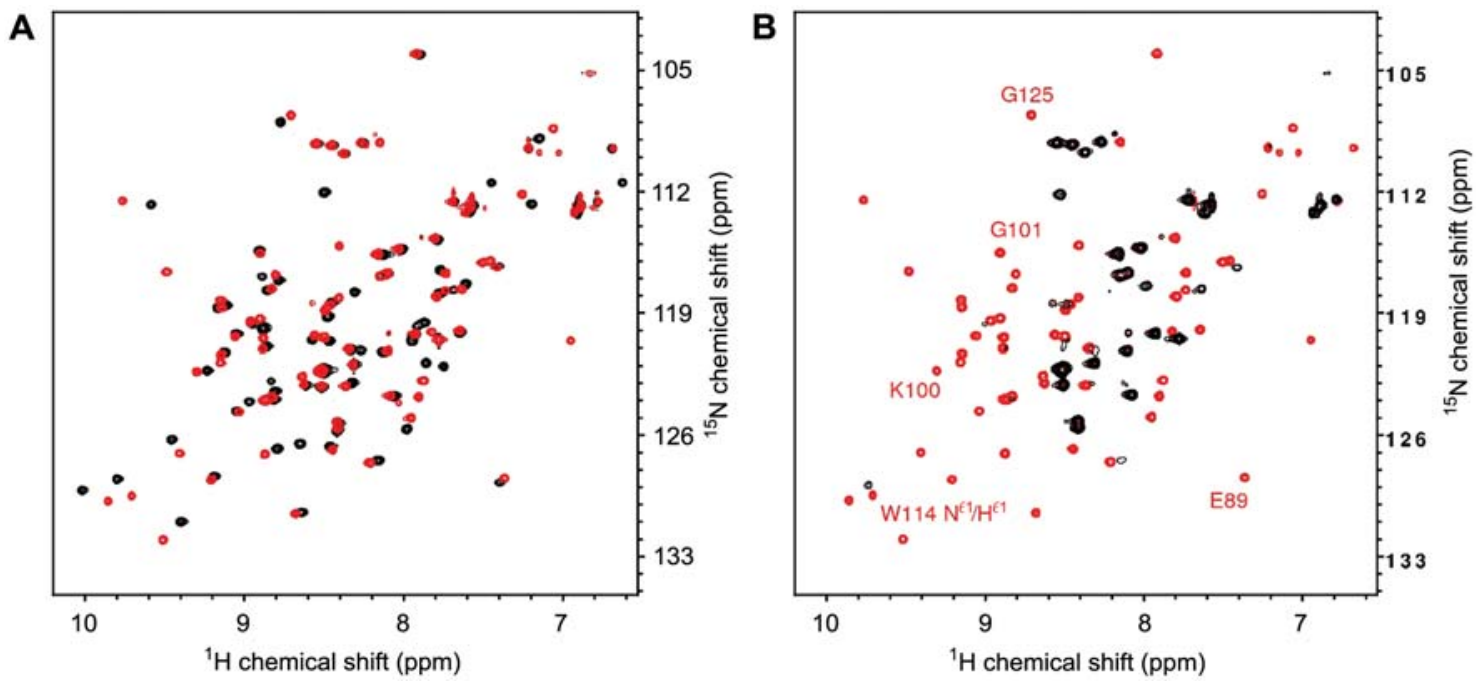

Figure $2{ }^{1} \mathrm{H}^{-15} \mathrm{~N}$ HSQC spectra of HckSH3 in the absence and presence of ligands.

All NMR spectra were recorded at $298 \mathrm{~K}$ on a Varian Unity INOVA spectrometer at a proton frequency of $600 \mathrm{MHz}$ with a Varian $\mathrm{XYZ}-\mathrm{PFG}-{ }^{1} \mathrm{H}\left\{{ }^{13} \mathrm{C},{ }^{15} \mathrm{~N}\right\}$ probe. Data were processed with NMRPipe (Delaglio et al., 1995) and analyzed with NMRView (Johnson, 2004) and CARA (Keller, 2004). All NMR samples contained uniformly ${ }^{15} \mathrm{~N}$-stable isotope-labeled HckSH3 in PBS buffer with $10 \%$ (v/v) deuterium oxide.

(A) PD1 peptide interacts with HckSH3. Superimposed ${ }^{1} \mathrm{H}-{ }^{15} \mathrm{~N}$ HSQC spectra of $0.26 \mathrm{~mm}$ HckSH3 in the absence (black contour lines) and presence (red contour lines) of equimolar PD1 peptide. During titration (data not shown), the black-colored peaks did not shift, but their intensities decreased with ongoing titration, and new peaks (red-colored) appeared. (B) PD1 peptide displaces Nef from HckSH3. Superimposed ${ }^{1} \mathrm{H}-{ }^{15} \mathrm{~N}$ HSQC spectra of $0.18 \mathrm{~mm}$ HckSH3. Both spectra show HckSH3 in the presence of $0.42 \mathrm{~mm}$ Nef, but are distinguished by the absence (black contour lines) and presence (red contour lines) of $1.1 \mathrm{~mm}$ PD1 peptide. During titration (data not shown), the black-colored peaks did not shift, but the intensities of some signals decreased and finally disappeared with ongoing titration, and new peaks (red-colored) appeared. Resonance signals used for quantitative evaluation are annotated. Complete resonance assignments are published elsewhere (Schmidt et al., 2007) and were deposited in BioMagResBank under BMRB entry number 6581.

dynamics for NMR (Grzesiek et al., 1996; Preusser et al., 2001). Furthermore, oligomerization of Nef was reported in NMR, crystallographic and cellular contexts (Kienzle et al., 1993; Fujii et al., 1996; Arold et al., 1997, 2000) and is required for a number of Nef functions (Liu et al., 2000), interestingly including Hck activation (Ye et al., 2004).

We titrated the peptide PD1 to the aforementioned Nef-HckSH3 sample. This resulted in displacement of Nef from HckSH3 and the formation of HckSH3-PD1 complexes (Figure 2B). The Nef-liganded HckSH3 NMR resonances decreased and eventually disappeared with increasing PD1 concentrations. Simultaneously, new well-dispersed resonances appeared that correspond to the PD1-liganded HckSH3, as a direct overlay of the resulting NMR spectrum (Figure $2 \mathrm{~B}$, red) and the PD1HckSH3 spectrum (Figure 2A, red) would clearly indicate (data not shown).

After correction for dilution effects, the signal intensities of the resonances in the NMR spectra can be quantitatively related to the concentration of the according moiety (e.g., PD1-liganded HckSH3). The data were successfully described by a model for competitive displacement of Nef from HckSH3, as shown in Figure 1B. Using $K_{\mathrm{d}}=0.23 \mu \mathrm{M}$ for PD1-HckSH3, as described above, the Nef-HckSH3 dissociation constant could be estimated as $0.44 \pm 0.03 \mu \mathrm{M}$. Both ligands contain the $\mathrm{SH} 3$ domain interaction PXXP motif, in accordance with the model of competition applied for the same binding site.

Dissociation constants for the interaction of HckSH3 domain with Nef proteins from different HIV-1 isolates are reported to be $0.25 \mu \mathrm{M}$ (Lee et al., 1995) and $0.6 \mu \mathrm{M}$
(Arold et al., 1998) and thus are in a similar range as our result. HckSH3 is regarded as the strongest ligand of Nef, and vice versa.

Compared to other data on $\mathrm{SH} 3$ domain interactions with PXXP peptide ligands, the interaction of Nef and Hck $\mathrm{SH} 3$ is regarded to be unusually strong (Renkema and Saksela, 2000). This is attributed to tertiary interactions between Nef and Hck SH3 (Lee et al., 1996; Arold et al., 1997). Here we present a peptide ligand that binds more strongly to Hck SH3 than Nef. This suggests that such strong SH3 ligand interaction can be obtained even without additional tertiary contacts. To the best of our knowledge, this is the first ligand to be described that allows displacement of Nef from an $\mathrm{SH} 3$ domain by competition for the PXXP binding site. This high-affinity peptide can be used for further in vitro or in vivo studies of Hck, the competition of Hck SH3-Nef interactions and as a lead for Hck-targeted drug design.

So far, antiviral drugs are directed against viral proteins. Such drugs, however, have the strong disadvantage that the virus can easily escape from the drug by mutations that lead to a decrease in affinity between the viral target protein and the drug. This is especially true for viruses with high mutation frequencies such as HIV. The solution to this problem might be a drug like PD1 that is directed against the host cellular target of the viral protein. Such a drug could actually shield the host cell protein against binding of the viral protein. As a consequence, the virus cannot escape from such a drug by single mutations within the viral genome, because such mutations cannot influence drug binding to the host cell 
protein. Regardless of potential side effects, this completely new concept of a 'molecular shield' for the host cell protein could eventually open a completely novel antiviral strategy.

\section{Acknowledgments}

This work was supported by a grant from Deutsche Forschungsgemeinschaft to D.W. (Wi 1472/4-2 and SFB575/B11).

\section{References}

Aiken, C., Konner, J., Landau, N.R., Lenburg, M.E., and Trono, D. (1994). Nef induces CD4 endocytosis: requirement for a critical dileucine motif in the membrane-proximal CD4 cytoplasmic domain. Cell 76, 853-864.

Arold, S., Franken, P., Strub, M.P., Hoh, F., Benichou, S., Benarous, R., and Dumas, C. (1997) The crystal structure of HIV1 Nef protein bound to the Fyn kinase $\mathrm{SH} 3$ domain suggests a role for this complex in altered $\mathrm{T}$ cell receptor signaling. Structure 5, 1361-1372.

Arold, S., O'Brien, R., Franken, P., Strub, M.P., Hoh, F., Dumas, C., and Ladbury, J.E. (1998). RT loop flexibility enhances the specificity of Src family SH3 domains for HIV-1 Nef. Biochemistry 37, 14683-14691.

Arold, S., Hoh, F., Domergue, S., Birck, C., Delsuc, M.A., Jullien, M., and Dumas, C. (2000) Characterization and molecular basis of the oligomeric structure of HIV-1 nef protein. Protein Sci. 9, 1137-1148.

Balter, M. (1996). HIV's other immune-system targets: macrophages. Science 274, 1464-1465.

Bell, I., Schaefer, T.M., Trible, R.P., Amedee, A., and Reinhart, T.A. (2001). Down-modulation of the costimulatory molecule, CD28, is a conserved activity of multiple SIV Nefs and is dependent on histidine 196 of Nef. Virology 283, 148-158.

Bodenhausen, G. and Ruben, D.J. (1980). Natural abundance N15 NMR by enhanced heteronuclear spectroscopy. Chem. Phys. Lett. 69, 185-189.

Briggs, S.D., Sharkey, M., Stevenson, M., and Smithgall, T.E. (1997). SH3-mediated Hck tyrosine kinase activation and fibroblast transformation by the Nef protein of HIV-1. J. Biol. Chem. 272, 17899-17902.

Craig, H.M., Pandori, M.W., Riggs, N.L., Richman, D.D., and Guatelli, J.C. (1999). Analysis of the SH3-binding region of HIV-1 nef: partial functional defects introduced by mutations in the polyproline helix and the hydrophobic pocket. Virology 262, 55-63.

Deacon, N.J., Tsykin, A., Solomon, A., Smith, K., Ludford-Menting, M., Hooker, D.J., McPhee, D.A., Greenway, A.L., Ellett, A., Chatfield, C., et al. (1995). Genomic structure of an attenuated quasi species of HIV-1 from a blood transfusion donor and recipients. Science 270, 988-991.

Delaglio, F., Grzesiek, S., Vuister, G.W., Zhu, G., Pfeifer, J., and Bax, A. (1995). NMRPipe: a multidimensional spectral processing system based on UNIX pipes. J. Biomol. NMR 6, 277-293.

Fujii, Y., Otake, K., Fujita, Y., Yamamoto, N., Nagai, Y., Tashiro, M., and Adachi, A. (1996). Clustered localization of oligomeric Nef protein of human immunodeficiency virus type 1 on the cell surface. FEBS Lett. 395, 257-261.

Garcia, J.V. and Miller, A.D. (1991). Serine phosphorylation-independent downregulation of cell-surface CD4 by nef. Nature 350, 508-511.

Greenberg, M.E., lafrate, A.J., and Skowronski, J. (1998). The SH3 domain-binding surface and an acidic motif in HIV-1 Nef regulate trafficking of class I MHC complexes. EMBO J. 17, 2777-2789.
Grzesiek, S., Bax, A., Clore, G.M., Gronenborn, A.M., Hu, J.S., Kaufman, J., Palmer, I., Stahl, S.J., and Wingfield, P.T. (1996). The solution structure of HIV-1 Nef reveals an unexpected fold and permits delineation of the binding surface for the $\mathrm{SH} 3$ domain of Hck tyrosine protein kinase. Nat. Struct. Biol. 3, 340-345.

Hrecka, K., Swigut, T., Schindler, M., Kirchhoff, F., and Skowronski, J. (2005). Nef proteins from diverse groups of primate lentiviruses downmodulate CXCR4 to inhibit migration to the chemokine stromal derived factor 1. J. Virol. 79, 10650-10659.

Johnson, B.A. (2004). Using NMRView to visualize and analyze the NMR spectra of macromolecules. Methods Mol. Biol. 278, 313-352.

Keller, R. (2004). The computer aided resonance assignment tutorial (Goldau, Switzerland: Cantina Verlag).

Kestler, H.W. III, Ringler, D.J., Mori, K., Panicali, D.L., Sehgal, P.K., Daniel, M.D., and Desrosiers, R.C. (1991). Importance of the nef gene for maintenance of high virus loads and for development of AIDS. Cell 65, 651-662.

Kienzle, N., Freund, J., Kalbitzer, H.R., and Mueller-Lantzsch, N. (1993). Oligomerization of the Nef protein from human immunodeficiency virus (HIV) type 1. Eur. J. Biochem. 214, 451-457.

Kirchhoff, F., Greenough, T.C., Brettler, D.B., Sullivan, J.L., and Desrosiers, R.C. (1995). Brief report: absence of intact nef sequences in a long-term survivor with nonprogressive HIV1 infection. N. Engl. J. Med. 332, 228-232.

Kohno, T., Kusunoki, H., Sato, K., and Wakamatsu, K. (1998). A new general method for the biosynthesis of stable isotopeenriched peptides using a decahistidine-tagged ubiquitin fusion system: an application to the production of mastoparan-X uniformly enriched with $15 \mathrm{~N}$ and $15 \mathrm{~N} / 13 \mathrm{C}$. J. Biomol. NMR 12, 109-121.

Lee, C.H., Leung, B., Lemmon, M.A., Zheng, J., Cowburn, D., Kuriyan, J., and Saksela, K. (1995). A single amino acid in the $\mathrm{SH} 3$ domain of Hck determines its high affinity and specificity in binding to HIV-1 Nef protein. EMBO J. 14, 5006-5015.

Lee, C.H., Saksela, K., Mirza, U.A., Chait, B.T., and Kuriyan, J. (1996). Crystal structure of the conserved core of HIV-1 Nef complexed with a Src family SH3 domain. Cell 85, 931-942.

Lepre, C.A., Moore, J.M., and Peng, J.W. (2004). Theory and applications of NMR-based screening in pharmaceutical research. Chem. Rev. 104, 3641-3676.

Liu, L.X., Heveker, N., Fackler, O.T., Arold, S., Le Gall, S., Janvier, K., Peterlin, B.M., Dumas, C., Schwartz, O., Benichou, S., and Benarous, R. (2000). Mutation of a conserved residue (D123) required for oligomerization of human immunodeficiency virus type 1 Nef protein abolishes interaction with human thioesterase and results in impairment of Nef biological functions. J. Virol. 74, 5310-5319.

Mangasarian, A., Piguet, V., Wang, J.K., Chen, Y.L., and Trono, D. (1999). Nef-induced CD4 and major histocompatibility complex class I (MHC-I) down-regulation are governed by distinct determinants: $\mathrm{N}$-terminal $\alpha$-helix and proline repeat of Nef selectively regulate MHC-I trafficking. J. Virol. 73, 1964-1973.

Moarefi, I., LaFevre-Bernt, M., Sicheri, F., Huse, M., Lee, C.H., Kuriyan, J., and Miller, W.T. (1997). Activation of the Src-family tyrosine kinase Hck by $\mathrm{SH} 3$ domain displacement. Nature 385, 650-653.

Preusser, A., Briese, L., Baur, A.S., and Willbold, D. (2001). Direct in vitro binding of full-length human immunodeficiency virus type 1 Nef protein to CD4 cytoplasmic domain. J. Virol. 75, 3960-3964.

Renkema, G.H. and Saksela, K. (2000). Interactions of HIV-1 NEF with cellular signal transducing proteins. Front. Biosci. 5, D268-283.

Saksela, K. (2004). Therapeutic targeting of interactions between Nef and host cell proteins. Curr. Drug Targets Immune Endocr. Metabol. Disord. 4, 315-319. 
Saksela, K., Cheng, G., and Baltimore, D. (1995). Proline-rich (PxxP) motifs in HIV-1 Nef bind to SH3 domains of a subset of Src kinases and are required for the enhanced growth of $\mathrm{Nef}^{+}$viruses but not for down-regulation of CD4. EMBO J. 14, 484-491.

Schaefer, T.M., Bell, I., Pfeifer, M.E., Ghosh, M., Trible, R.P., Fuller, C.L., Ashman, C., and Reinhart, T.A. (2002). The conserved process of TCR/CD3 complex down-modulation by SIV Nef is mediated by the central core, not endocytic motifs. Virology 302, 106-122.

Schindler, M., Munch, J., Kutsch, O., Li, H., Santiago, M.L., Bibollet-Ruche, F., Muller-Trutwin, M.C., Novembre, F.J., Peeters, M., Courgnaud, V., et al. (2006). Nef-mediated suppression of $\mathrm{T}$ cell activation was lost in a lentiviral lineage that gave rise to HIV-1. Cell 125, 1055-1067.

Schmidt, H., Hoffmann, S., Tran, T., Stoldt, M., Stangler, T., Wiesehan, K., and Willbold, D. (2007). Solution structure of a Hck $\mathrm{SH} 3$ domain ligand complex reveals novel interaction modes. J. Mol. Biol. 365, 1517-1532.

Stangler, T., Hartmann, R., Willbold, D., and König, B.W. (2006). Modern high resolution NMR for the study of structure, dynamics and interactions of biological macromolecules. $Z$. Phys. Chem. 220, 567-613.

Stove, V., Van de Walle, I., Naessens, E., Coene, E., Stove, C., Plum, J., and Verhasselt, B. (2005). Human immunodeficiency virus Nef induces rapid internalization of the T-cell coreceptor CD8 $\alpha \beta$. J. Virol. 79, 11422-11433.
Swigut, T., Shohdy, N., and Skowronski, J. (2001). Mechanism for down-regulation of CD28 by Nef. EMBO J. 20, 1593-1604.

Tran, T., Hoffmann, S., Wiesehan, K., Jonas, E., Luge, C., Aladag, A., and Willbold, D. (2005). Insights into human Lck SH3 domain binding specificity: different binding modes of artificial and native ligands. Biochemistry 44, 15042-15052.

Trible, R., Emert-Sedlak, L., and Smithgall, T. (2006). HIV-1 Nef selectively activates SRC family kinases HCK, LYN, and C$\mathrm{SRC}$ through direct $\mathrm{SH} 3$ domain interaction. J. Biol. Chem. 281, 27029-27038.

Wang, Z.X. (1995). An exact mathematical expression for describing competitive binding of two different ligands to a protein molecule. FEBS Lett. 360, 111-114.

Welker, R., Kottler, H., Kalbitzer, H.R., and Krausslich, H.G. (1996). Human immunodeficiency virus type 1 Nef protein is incorporated into virus particles and specifically cleaved by the viral proteinase. Virology 219, 228-236.

Ye, H., Choi, H., Poe, J., and Smithgall, T.E. (2004). Oligomerization is required for HIV-1 Nef-induced activation of the Src family protein-tyrosine kinase, Hck. Biochemistry 43, 15775-15784.

Received January 26, 2007; accepted March 20, 2007 